\title{
RX And: An intermediate between Z Cam and VY Scl stars
}

\author{
M. R. Schreiber ${ }^{1}$, B. T. Gänsicke ${ }^{1,2}$, and J. A. Mattei ${ }^{3}$ \\ 1 Universitäts-Sternwarte, Geismarlandstr.11, 37083 Göttingen, Germany \\ 2 Department of Physics and Astronomy, University of Southampton, Highfield, Southampton SO17 1BJ, UK \\ 3 American Association of Variable Star Observers, 25 Birch Street, Cambridge, MA 02138, USA
}

Received 2 January 2002 / Accepted 22 January 2002

\begin{abstract}
We present the AAVSO long term visual light curve of RX And. The system has been classified as a Z Cam-type dwarf nova, showing alternating standstills and dwarf nova outbursts. Our data reveal that RX And has also characteristics of a VYScl star, remaining for extended periods in a low state $\sim 3.5$ mag fainter than the standstill with no detectable outbursts. We discuss this result in the context of mass transfer variations in CVs and the still poorly understood VY Scl phenomenon.
\end{abstract}

Key words. accretion, accretion discs - binaries: close - stars: individual: RX And - novae, cataclysmic variables

\section{Introduction}

In cataclysmic variables (CVs) (Warner 1995, for an encyclopaedic review) a white dwarf accretes material from its Roche-lobe filling secondary, a low mass main-sequence star. A significant fraction of CVs shows phases of low luminosity in their long term light curves. Whereas these low states appear to be a general phenomenon among the magnetic and discless AMHer systems, only a relatively small group of non-magnetic CVs shows low states: the VY Scl stars, a subclass of the high $\dot{M}$ novalike variables, which is defined by the low states of its members.

Almost all VYScl stars have orbital periods in the range of $3 \mathrm{hr}<P_{\text {orb }}<4 \mathrm{hr}$ (e.g. Shafter 1992), just above the $2 \mathrm{hr}<P_{\text {orb }}<3 \mathrm{hr}$ period gap. Considering in addition the deficiency of dwarf novae with $3 \mathrm{hr}<P_{\text {orb }}<4 \mathrm{hr}$ (Shafter 1992; Hellier \& Naylor 1998) it appears tempting to interpret the VY Scl phenomenon as an evolutionary effect. Livio \& Pringle (1994) suggested that dwarf novae, evolving towards shorter orbital periods, turn into VY Scl stars because of increased stellar activity on the secondary. In this scenario star spots intermittently cover the $L_{1}$ point and - reducing the mass transfer - are the cause of the low states. Eventually, the mass transfer completely stops when the system reaches the upper boundary of the period gap. However, King \& Kolb (1995) showed that the standard explanation for the period gap, i.e. interrupted magnetic braking (Verbunt \& Zwaan 1981; Rappaport et al. 1983; Kolb 1993), is much more plausible.

Send offprint requests to: M. R. Schreiber, e-mail: mschrei@uni-sw.gwdg.de
More recently, Hellier \& Naylor (1998) argued against an evolutionary connection between dwarf novae and VY Scl stars as no transitional systems between the Z Cam and VY Scl subtype are known. Following Warner (1995), they claim that VY Scl stars and Z Cam systems are distinctly different types of CVs.

In this paper we present the long term light curve of RX And and show that this star has to be classified as both, a Z Cam and a VY Scl system, and discuss the implications of this result.

\section{RX And and low-states in CVs}

Figure 1 shows the AAVSO long term light curve of RX And, a $P_{\text {orb }}=5.04 \mathrm{hr} \mathrm{CV}$ which has so far been classified as a Z Cam system. Indeed, the system repeatedly shows picture-book Z Cam-behaviour, e.g. in the periods MJD = 46300-46500, 50600-50900, or 51300-51500. However, inspecting Fig. 1 in more detail it becomes apparent that RX And shows large variations of its outburst behaviour, i.e. periods of frequent short low-amplitude outbursts (42700-42900), irregular inactive states, possibly with mini-outburst activity (43300-43600) and typical long dwarf nova outbursts interrupted by "disrhythmia" (48500-48700).

At MJD $=50300-50500$ RX And was found in a deep low state, $V \approx 15.2$, much fainter than the typical quiescent level of $V \approx 14$. In Fig. 2 we compare the deep low state of RX And with the low states observed in the typical VY Scl star TT Ari and in the magnetic CV AM Her. A characteristic common to all three systems is that their 


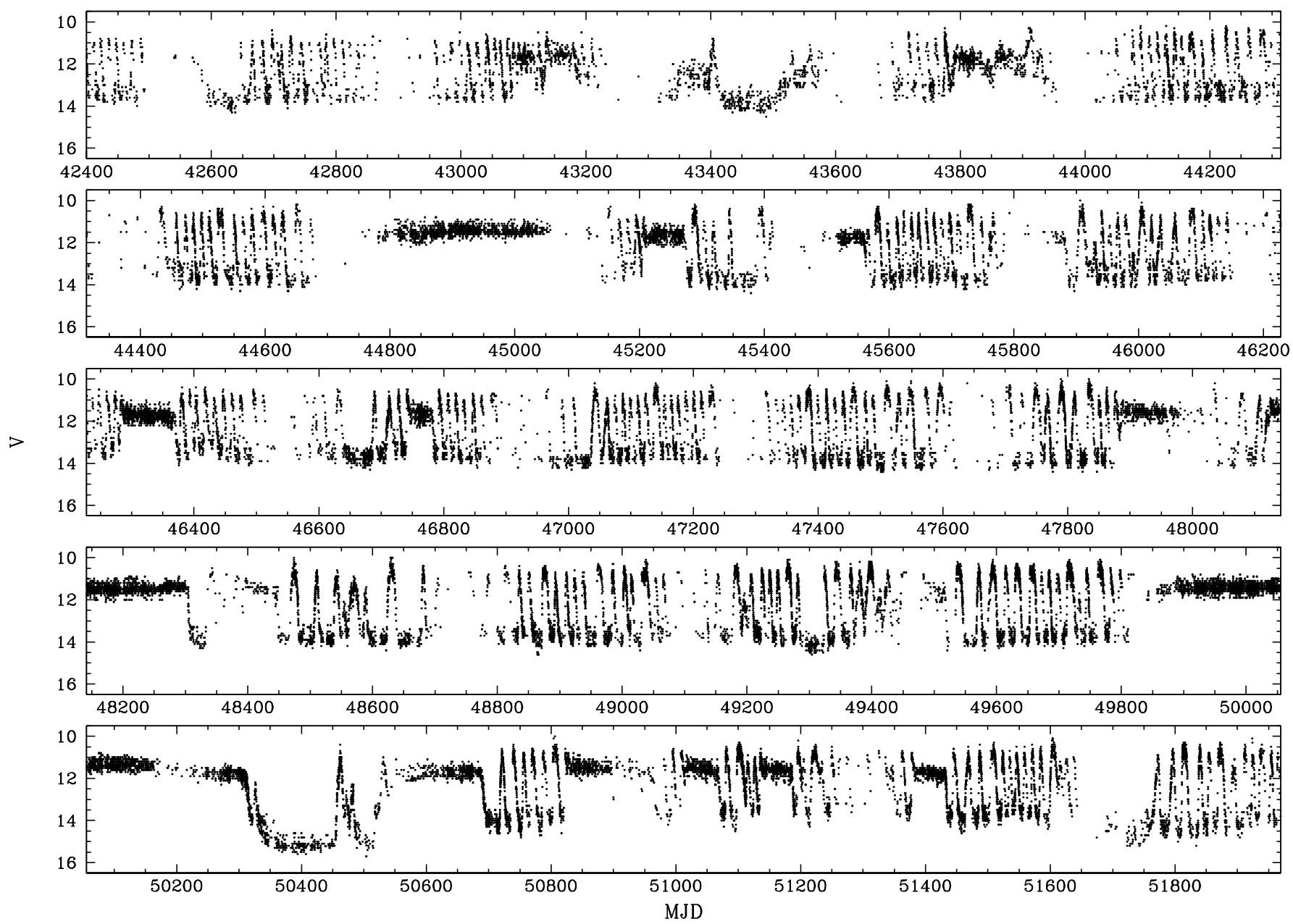

Fig. 1. The long term light curve of RX And. The system shows a variety of outburst behaviours ranging from typical Z Cam behaviour (e.g. 46 300-46 500), periods of frequent short low-amplitude outbursts (42 700-42 900), irregular low states (43 300-43 600), and typical long dwarf nova outbursts interrupted by "disrhythmia" (48500-48 700) to typical deep VY Scl low-states (50 300-50 500).

optical and ultraviolet low state spectra are almost entirely dominated by the photospheric emission from the secondary star and from the white dwarf, with very little contribution from the accretion disc/stream (TT Ari: Shafter et al. 1985; Gänsicke et al. 1999, RX And: Sion et al. 2001, AM Her: Gänsicke et al. 1995). While the amplitude of the low states in RX And ( $\left.V_{\text {low state }}-V_{\text {standstill }}\right)$ and in TT Ari ( $\left.V_{\text {low state }}-V_{\text {high state }}\right)$ are very similar, $\Delta V \approx 3.5$, the low state in AMHer has a significantly lower amplitude, $V_{\text {low state }}-V_{\text {high state }} \approx 2$. This can be easily understood considering that the non-magnetic disc systems RX And and TT Ari emit a larger fraction of the released gravitational energy at optical wavelength than the magnetic and discless system AM Her. While the three low states differ in duration (RX And: $\sim 100 \mathrm{~d}$, AM Her: $\sim 300$ d, TT Ari: $730 \mathrm{~d}$ ), the overall morphology of the low state light curves is very similar in all three systems ${ }^{1}$. As a consequence of the deep low state observed in RX And,

\footnotetext{
${ }^{1}$ It is interesting to note that all three systems show a precursor brightening shortly before switching again to their typical high state/standstill brightness.
}

the system has to be classified not only a Z Cam system but also as a VY Scl star.

It is worth noting that in both, the AMHer and the VY Scl class, the amplitude and duration of the low states strongly vary between the members of the class. While the VY Scl star TT Ari (Fig. 2, left) suffered a single but deep and long-lasting low state during the last 25 years, MV Lyr - another member of the VY Scl class - varies frequently through a wide range of visual brightness states (Rosino et al. 1993). Among the magnetic CVs, the only system with a detailed long term visual light curve is AMHer (Fig. 2, right). However, a number of other magnetic CVs have been observed frequently enough at optical, ultraviolet or X-ray wavelengths to establish at least a rough estimate of their long term variability. For instance, QQ Vul has never been observed in a low state, and EF Eri has been found to be in a high state for almost twenty years after its discovery, whereafter it switched off in 1997 and remains in deep low state until today.

Considering the common characteristics of the low states observed in AM Her and VY Scl stars, and now also 

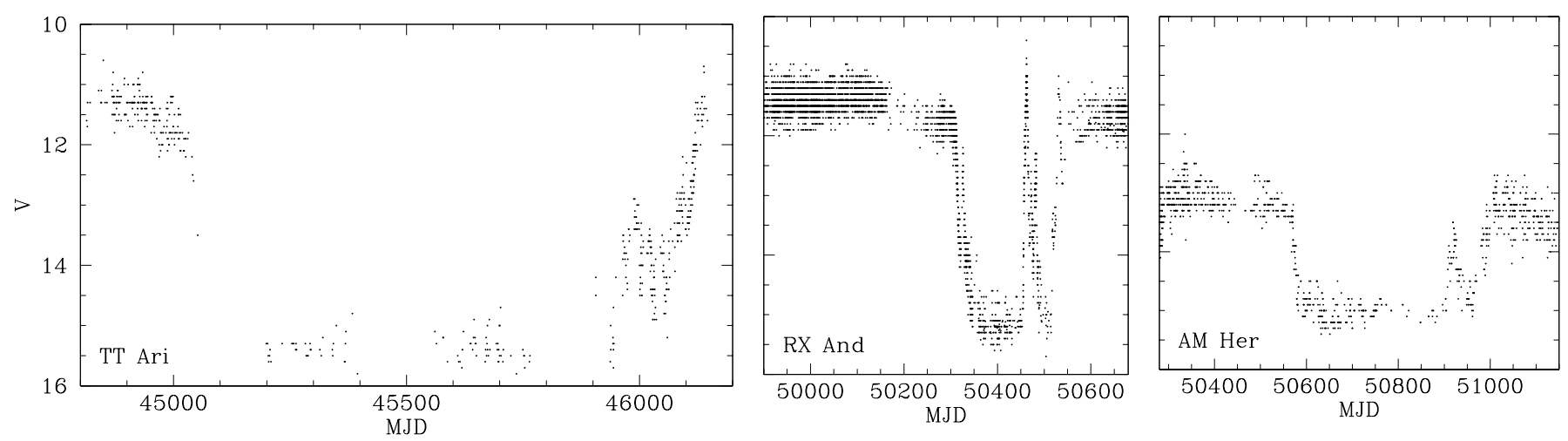

Fig. 2. Low states in CVs. From left to right: the VY Scl star TT Ari, the Z Cam type dwarf nova RX And, and the magnetic system AM Her. Note that all three light curves are shown on the same scale.

in the dwarf nova RX And, it appears plausible that the low states in all these systems are caused by the same phenomenon: strong and irregular variations of the mass transfer rate from the secondary star.

\section{Mass transfer variations and disc instabilities}

In AM Her systems, where the mass transfer rate from the secondary equals the mass accretion rate every moment, the only plausible origin of the low states are drops in the mass transfer rate. In contrast to that, it has been shown that the disc instability model (DIM) in its current form (see Lasota 2001, for a recent review) cannot explain the VYScl phenomenon with mass transfer variations alone, as the model predicts small outbursts even if the mass transfer ceases completely (King \& Cannizzo 1998; Schreiber et al. 2000; Buat-Ménard et al. 2001). However, it is clear that the occurrence of low states is correlated with significantly decreased mass transfer.

Buat-Ménard et al. (2001) showed that the transition between standstill and regular outbursts in Z Cam systems can be explained within the DIM by only moderate $(\sim 30 \%)$ variations of the mass transfer rate when considering stream impact heating of the outer disc. Their calculations show that decreases in the mass transfer rate of $\sim 1-2$ orders of magnitude, such as observed, e.g., in AM Her (Hessman et al. 2000), result in a decrease of the outburst amplitude by $\sim 2$ mag. Buat-Ménard et al. argued that such variations of the outburst magnitude are not observed, and, thus, categorically excluded the occurrence of large mass transfer variations in dwarf novae.

Apparently, RX And is a dwarf nova that does show significant variations in its outburst amplitude (compare MJD $\approx 42700$ and 49700 in Fig. 1) ${ }^{2}$. While low-amplitude ( $\lesssim 1 \mathrm{mag}$ ) outbursts may still occur during the intermediate brightness state $(43400)$, the two stellar components dominate the emission during the deep low state (50 400).

\footnotetext{
${ }^{2}$ Notice, contrary to calculated light curves (e.g. BuatMénard et al. 2001) the observed low-amplitude outbursts (MJD $\approx 42700$ ) tend to have shorter recurrence times than the large-amplitude outbursts (MJD $\approx 49700$ ).
}

Thus, RX And appears to be a dwarf nova system with strong mass transfer variations.

\section{Discussion}

The deep low state detected in the long term light curve of RX And shows that this system is a VY Scl as well as a Z Cam star. Thus the answer to the question of Hellier \& Naylor (1998) "And why is there no transitional object between a $Z C$ and a $V Y$ ?" is: such objects do exist! Moreover, one has to conclude that Z Cam and VY Scl stars are obviously not distinctly different systems although dwarf nova outbursts have a different physical origin (disc instabilities) than the typical VY Scl variability (strong mass transfer variations). One is prone to speculate whether Z Cam stars do in fact evolve into VY Scl systems, and whether RXAnd with its orbital period of $5.04 \mathrm{hr}$ is just at the beginning of its metamorphosis.

The variegated light curve of RX And (Fig. 1) strongly suggests the presence of significant mass transfer variations in the system, and we suggest a sharp drop in the mass transfer rate as the common origin for the low states observed in RX And, TT Ari, and AM Her (Fig. 2). Whether the occurrence of strong mass transfer variations is an evolutionary effect that peaks in the VY Scl regime, $3 \mathrm{hr}<P_{\text {orb }}<4 \mathrm{hr}$, is still an open question. There exists some observational evidence that mass transfer variations are - to some extent - present in all CVs: high/low state transitions have been observed in most of the magnetic AMHer stars, which have no accretion disc acting as a mass buffer, and also the well-studied SU UMa dwarf nova HT Cas was found several times in a low state significantly fainter than the usual quiescent level (Robertson \& Honeycutt 1996; Wood et al. 1995).

We advocate that not the occurence of mass transfer variations, but the impact that such mass transfer variations have on the observed long term light curve of non-magnetic CVs might be related to an evolutionary effect. Indeed, Schreiber et al. (2000) have shown that the timescale on which the accretion disc reacts on mass transfer variations increases with the disc mass, i.e. with longer 
orbital periods. In this scenario, mass transfer variations are the origin of irregularities in the long term light curves of a variety of dwarf novae above the period gap, low states during quiescence in disc systems below the gap, the VYScl phenomenon, and the high/low state transitions of AM Her stars at all orbital periods.

Clearly, our understanding of the relation between dwarf novae and VY Scl stars in particular, and the origin of strong mass transfer variations in $\mathrm{CVs}$ in general would greatly benefit from a larger sample of CVs with high quality long term light curves.

Acknowledgements. We thank the AAVSO observers worldwide for making this research possible. MRS and BTG were supported from the DLR under grant 50 OR 99036 . BTG also acknowledges support by a PPARC Advanced Fellowship.

\section{References}

Buat-Ménard, V., Hameury, J.-M., \& Lasota, J.-P. 2001, A\&A, 369, 925

Gänsicke, B. T., Beuermann, K., \& de Martino, D. 1995, A\&A, 303, 127
Gänsicke, B. T., Sion, E. M., Beuermann, K., et al. 1999, A\&A, 347,178

Hellier, C., \& Naylor, T. 1998, MNRAS, 295, L50

Hessman, F. V., Gänsicke, B. T., \& Mattei, J. A. 2000, A\&A, 361,952

King, A. R., \& Cannizzo, J. K. 1998, ApJ, 499, 348

King, A. R., \& Kolb, U. 1995, ApJ, 439, 330

Kolb, U. 1993, A\&A, 271, 149

Lasota, J.-P. 2001, New Astron. Rev., 45, 449

Livio, M., \& Pringle, J. E. 1994, ApJ, 427, 956

Rappaport, S., Joss, P. C., \& Verbunt, F. 1983, ApJ, 275, 713

Robertson, J. W., \& Honeycutt, R. K. 1996, AJ, 112, 2248

Rosino, L., Romano, G., \& Marziani, P. 1993, PASP, 105, 51

Schreiber, M. R., Gänsicke, B. T., \& Hessman, F. V. 2000, A\&A, 358, 221

Shafter, A. W. 1992, ApJ, 394, 268

Shafter, A. W., Szkody, P., Liebert, J., et al. 1985, ApJ, 290, 707

Sion, E. M., Szkody, P., Gaensicke, B., et al. 2001, ApJ, 555, 834

Verbunt, F., \& Zwaan, C. 1981, A\&A, 100, L7

Warner, B. 1995, Cataclysmic Variable Stars (Cambridge: Cambridge University Press)

Wood, J. H., Naylor, T., Hassall, B. J. M., \& Ramseyer, T. F. 1995, MNRAS, 273, 772 